\title{
A Predictive Neural Network-Based Cascade Control for pH Reactors
}

\author{
Mujahed AlDhaifallah, ${ }^{1}$ Shebel Alsabbah, ${ }^{1}$ and Iqbal Mujtaba ${ }^{2}$ \\ ${ }^{1}$ College of Engineering at Wadi Aldawaser, Prince Sattam bin Abdulaziz University, P.O. Box 54, Wadi Aldawaser 11991, Saudi Arabia \\ ${ }^{2}$ Chemical Engineering Department, School of Engineering, University of Bradford, Bradford, West Yorkshire BD7 IDP, UK \\ Correspondence should be addressed to Mujahed AlDhaifallah; muja2007hed@gmail.com
}

Received 8 May 2016; Revised 7 July 2016; Accepted 25 July 2016

Academic Editor: Qingsong Xu

Copyright (C) 2016 Mujahed AlDhaifallah et al. This is an open access article distributed under the Creative Commons Attribution License, which permits unrestricted use, distribution, and reproduction in any medium, provided the original work is properly cited.

\begin{abstract}
This paper is concerned with the development of predictive neural network-based cascade control for $\mathrm{pH}$ reactors. The cascade structure consists of a master control loop (fuzzy proportional-integral) and a slave one (predictive neural network). The master loop is chosen to be more accurate but slower than the slave one. The strong features found in cascade structure have been added to the inherent features in model predictive neural network. The neural network is used to alleviate modeling difficulties found with $\mathrm{pH}$ reactor and to predict its behavior. The parameters of predictive algorithm are determined using an optimization algorithm. The effectiveness and feasibility of the proposed design have been demonstrated using MatLab.
\end{abstract}

\section{Introduction}

The predictive neural network control (PNNC) is a straightforward application of neural networks to nonlinear control [1-3]. It is a combination of the main features of neural network (NN) with model predictive control (MPC). MPNNC is applied to industrial processes which usually contain extreme nonlinear dynamics. It aims to predict the behavior of the process with the existence of moving reference trajectory and disturbances by minimizing a certain cost function that takes into account the future output prediction errors [4].

The classical feedback control structures are not very efficient with industrial applications that contain complex nonlinearities. Hence, to enhance the stability of the controlled plant at different reference trajectories and to modify the dynamic features by reducing the settling time, a cascade control structure could be developed with a slave control loop faster but less accurate than the master one [5,6]. This control scheme would modify the overall performance of the controlled plant when comparing with the conventional control structures.

The chosen case study in this paper is the $\mathrm{pH}$ reactors. The difficulty of controlling $\mathrm{pH}$ processes resides in the nonlinear dynamics and parameter uncertainties of such processes. This behavior is due to the logarithmic relationship between the hydrogen ions concentrations $\left[\mathrm{H}^{+}\right]$and the $\mathrm{pH}$ value [1]

This paper illustrates feasible control of the $\mathrm{pH}$ titration plant using predictive and intelligent-based techniques. It also investigates the performance of predictive neural network controller in cascaded structure against the more traditional single loop fuzzy PI controllers. As conclusion, the proposed cascaded structure and the MPNN controller have outperformed the conventional ones. Simulation results have been obtained using MatLab/Simulink. These results demonstrate the feasibility and effectiveness of the proposed design.

This paper is structured as follows: in Section 2 the theoretical background of classical, neural network-based model predictive control algorithms and the cascade control structure are presented. In Section 3 the modeling and control of $\mathrm{pH}$ reactor are represented as a case study. Performance of the proposed controller, interpretation of results, and conclusions are presented in Sections 4, 5, and 6.

\section{Preliminaries}

2.1. Neural Network-Based Modeling and Control. To overcome the difficulties in the mathematical modeling of $\mathrm{pH}$ reactors in titration processes, many techniques would be 


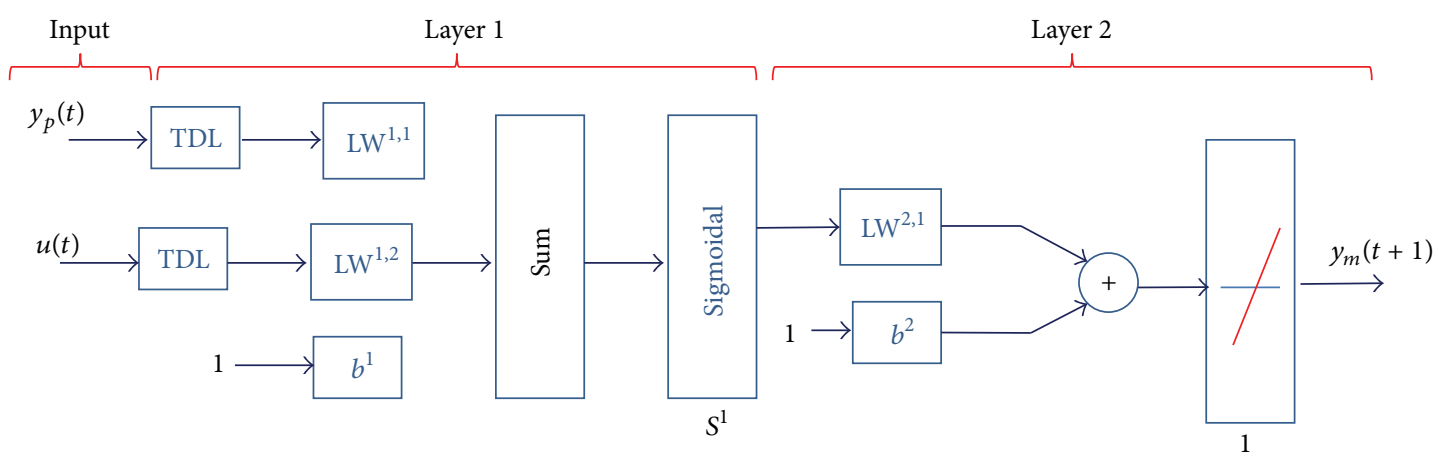

FIGURE 1: NN model of $\mathrm{pH}$ reactor.

used and have the capabilities to meet such demand. In our case, a data-based predictive neural network model is utilized.

Neural networks have been increasingly used in the chemical process industry [7], especially for modeling and control of some complex nonlinear processes where process understanding is limited [5]. For example, in the 90s of the last century, many researchers have explored the integration of process knowledge into black box models to provide "grey box" or "hybrid" models $[8,9]$. Over the years, there has been a tremendous amount of research focusing on modeling and control of $\mathrm{pH}$ chemical reactors with different control strategies [7-11]. A few of these researches undertaken over the past 5 years include nonlinear-based control; fuzzy control with genetic algorithm; adaptive heuristic control; PID-fuzzy control; and nonlinear generalized predictive control [7].

In this paper, a predictive neural network (PNN) model based on the experimental data is developed to study the performance of $\mathrm{pH}$ reactors in titration processes under different operating parameters such as the fluctuation in feed flow rate of acid and nonstationary $\mathrm{pH}$ setpoint. The proposed model was found to be capable of predicting accurately the unseen data of the titration process. The prediction error between the NN predictions and experimental data was found to validate our approach, as will be seen later.

The three steps involved in the development of NN model are the following:

(a) Generation of input-output data to train the network: these data should contain all the relevant information about the dynamics of $\mathrm{pH}$ reactors. The input was given to the conventional model of the $\mathrm{pH}$ reactor and from the conventional model, the input and output were sampled for 0.02 sampling instants and the required sampled data are obtained to train the network.

(b) The sigmoid activation function of a feed forward network was selected based on the trials with different structures of multilayer perceptron.

The NN topology selected here consists of 4 neurons in the input layer, 7 neurons in the hidden layer, and one neuron in the output layer. Hence it is selected as optimal architecture of NN (lowest error), Figure 1. The back propagation algorithm is used in
TABLE 1: NN parameters for modeling of $\mathrm{pH}$ reactor.

\begin{tabular}{lc}
\hline Parameters & Values \\
\hline Input neurons & 4 \\
output neurons & 1 \\
Hidden layer neurons & 7 \\
Number of hidden layer & 7 \\
Activation function & Sigmoidal \\
Training algorithm & Levenberg-Marquardt \\
Iteration & 10000 \\
Architecture & Feedforward \\
Initial weights & 1 \\
\hline
\end{tabular}

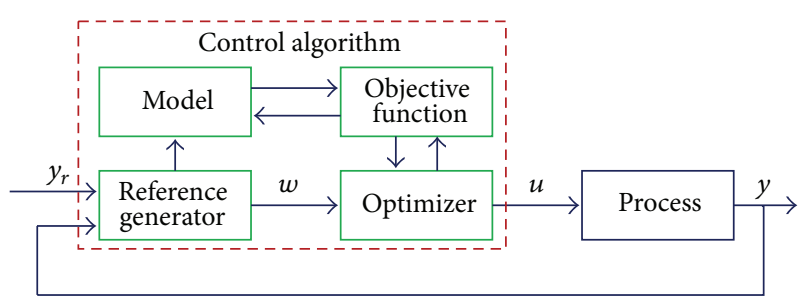

FIgURE 2: The scheme of classical MPC.

the modeling of $\mathrm{pH}$ reactor as a training algorithm. Before training the process weights are initialized to small random numbers. The weights are adjusted till error gets minimized for all training sets. When the error for the entire set is acceptably low, the training is stopped. Table 1 shows the parameters used in developing the $\mathrm{NN}$-based model for the $\mathrm{pH}$ reactor.

(c) Model validation: the final step is the validation. It aims to evaluate the model performance using training and test data. The input and target were presented to the network and the network was trained using Levenberg-Marquardt algorithm.

2.2. Classical Model-Based Predictive Control (MPC). The scheme of classical MPC is shown in Figure 2. It consists of the plant model and the optimization block. It is one of several different model-based control techniques that are associated with the same idea, as it is. 
Its target is to predict the future behavior of the process over a certain time horizon using the dynamic model and obtaining the control actions to minimize a certain criterion:

$$
\begin{aligned}
J(k, u(k))= & \sum_{j=N 1}^{N 2}\left(y(k+j)-y_{r}(k+j)\right)^{2} \\
& +\lambda \sum_{j=1}^{N_{u}}(u(k+j-1))^{2},
\end{aligned}
$$

where $y(k+j)$ is process output, $y_{r}(k+j)$ is reference trajectory, and $u(k+j)$ is the control output. It presents the $j$ step ahead predictions. $N_{1}$ and $N_{2}$ are the limits prediction horizon of the controlled variable. $N_{u}$ is the prediction horizon of the reference signal; it is used to reduce the computational load of the method. $\lambda$ is the weight of the control output [1].

Only the first control signal of the calculated sequence is applied to the controlled process at each sampling period. At the next sampling time the procedure is repeated. This is known as the receding horizon concept $[1,2]$.

The process input and output constraints are as given in

$$
\left(\begin{array}{c}
u_{\min } \leq u \leq u_{\max } \\
\Delta u_{\min } \leq \Delta u \leq \Delta u_{\max } \\
y_{\min } \leq y \leq y_{\max } \\
\Delta y_{\min } \leq \Delta y \leq \Delta y_{\max }
\end{array}\right)
$$

MPC algorithms are considered to be robust ones in industrial control applications because of their ability to handle constraints.

2.3. Model Predictive Neural Network Control (MPNNC). Figure 3 shows the neural network (NN) as a function approximator. It has been applied very successfully in the identification and control of dynamic systems [5]. The unknown function corresponds to a controlled plant, and the neural network is the identified plant model.

The difference between plant output and the NN output is the prediction error which is used as the NN training signal. NN uses previous inputs/outputs to predict future values of the plant output.

Levenberg-Marquardt (LM) algorithm is used as an iterative technique to train the net. It locates the minimum of a function that is expressed as the sum of squares of nonlinear functions. The NN can be trained offline in batch mode, using data collected from the operation of the plant [1].

2.4. Cascade Control Structure. Cascade control is a multiloop scheme commonly used in chemical plants [6] that can improve control performance of a system, when disturbances affect the control input. It consists of outer (master, C1) and inner (slave, C2) loop as shown in Figure 4. Inner and outer control loops are formed each with an individual feedback controller. The major benefit from using cascade control is that disturbances arising within the secondary loop are corrected by the secondary controller before they can affect the value of the primary controlled output.

The slave controller (C2) is tuned from model parameters (P2), while the master controller (C1) is tuned with the inner loop in operation from model parameters (P1).

\section{Case Study}

$\mathrm{pH}$ reactor system (Figure 5) is a typical chemical reactor system with extremely nonlinear dynamics. It is one of the plants that submit challenges to scientist and engineers because of the lack of understanding the dynamics of such process and the difficulty to develop a suitable control strategy.

3.1. Modeling of Chemical Reactor. The $\mathrm{pH}$ reactor has two input continuously flowing streams (base and acid), and this continuity of the process gives the titration a special theme, in which the dynamics of the titrated streams are involved. Referring to literature in modeling and dynamics of $\mathrm{pH}$ reactors [11-13], the following equation is derived:

$$
\mathrm{pH}=\log _{10}\left[\frac{-\left(k Q_{a} C_{a} /\left(Q_{a}+Q_{b}\right)-Q_{b} C_{b} /\left(Q_{a}+Q_{b}\right)\right)+\sqrt{\left(k Q_{a} C_{a} /\left(Q_{a}+Q_{b}\right)-Q_{b} C_{b} /\left(Q_{a}+Q_{b}\right)\right)^{2}-4\left(10^{-14}\right)(-1)}}{2\left(10^{-14}\right)}\right],
$$

where $C_{a}$ and $C_{b}$ are the values of concentration for acid and base streams, respectively; $Q_{a}$ and $Q_{b}$ are their volumetric flow rates, respectively, and $k$ is a constant that depends on the strength of acid entering the reactor $(k=1$, for strong acid-strong base system).

For simulation purposes, we would consider the mixer is an ideal continuous stirred tank reactor CSTR. The $\mathrm{pH}$ model of (3) pretends that the water is at the ideal temperature. Moreover, it is assumed that composition conditions which make the ion product exactly $10^{14}$, the concentrations of the acid and base, flow rates of the water, and value for the acid dissociation constant are known.

The titration curve, shown in Figure 6, shows clearly the direct S-relation between the $\mathrm{pH}$ value as a process output and the flow of base as a control output. So, $\mathrm{pH}$ is considered as the master process variable to be controlled that is affected directly by $Q_{b}$ as a manipulated variable.

The specifications of the considered $\mathrm{pH}$ model are that the strong acid (HCL) is flowing in constant value $(2 \mathrm{~mL} / \mathrm{sec})$, its concentration is equal to $0.95 \mathrm{~mol} / \mathrm{L}$, the process variable will 


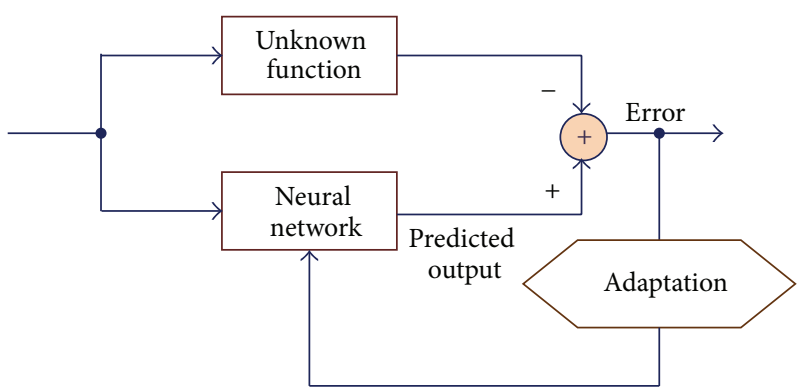

FIGURE 3: NN as a function approximator.

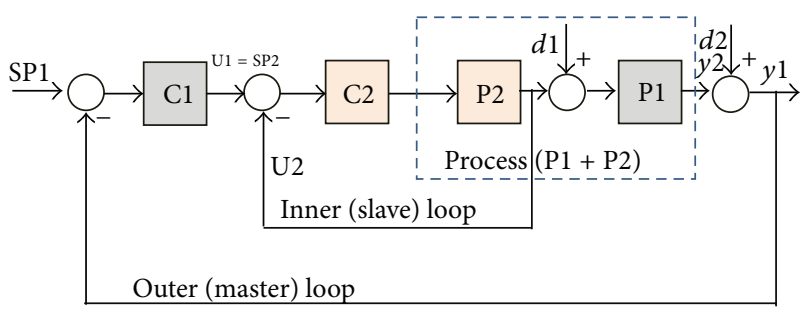

Figure 4: Cascade block diagram.

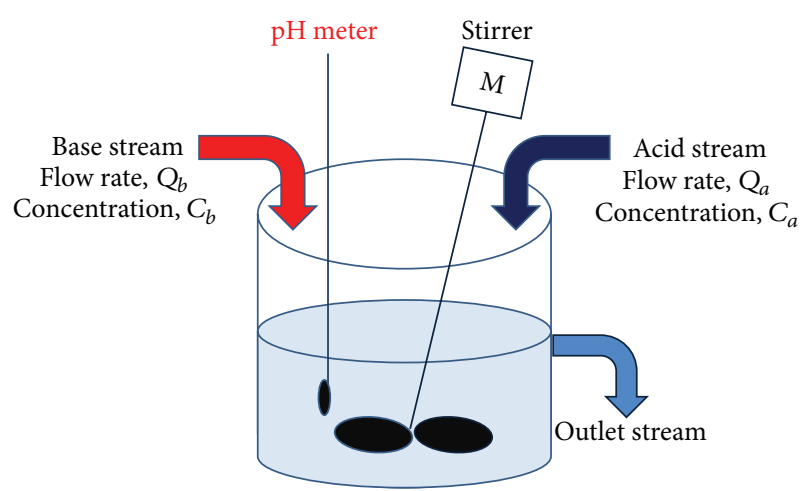

FIGURE 5: Schematic diagram $\mathrm{pH}$ reactor.

be the flow of strong base $(\mathrm{NaOH})$, and its concentration is $1.9 \mathrm{~mol} / \mathrm{L}$.

3.2. Cascade Control of the $p H$ Reactor. The designed structure is the cascade one (Figure 7), where the outer fuzzy PIbased control loop $(\mathrm{Cl})$ is chosen to enhance stability at the final state, and the MPNNC (C2) is considered as an inner loop to improve dynamics by minimization of over/under shoots area and fasten the settling time.

Also, $\mathrm{Cl}$ is the outer (master) controller, $\mathrm{C} 2$ is inner (slave) controller, ADC is the analog to digital converter, DAC is the digital to analog converter, $P / I$ is the pressure (3-15 psi) to current $(4-20 \mathrm{~mA})$ converter, $\mathrm{F}_{\mathrm{T}} \mathrm{T}$ is the flow out transmitter of the product, $\mathrm{F}_{\mathrm{T}} \mathrm{C}$ is the flow out controller of the product, $\mathrm{pHT}$ is $\mathrm{pH}$ transmitter, $\mathrm{T}_{\mathrm{S}} \mathrm{T}$ is the temperature transmitter of steam in, $\mathrm{F}_{\mathrm{B}} \mathrm{CV}_{\mathrm{i}}$ is the automatic control valve on the base stream, $\mathrm{F}_{\mathrm{A}} \mathrm{CV}_{\mathrm{i}}$ is a manual-actuated control valve on the acid stream, $\mathrm{T}_{\mathrm{S}} C V_{\mathrm{i}}$ is the control valve on the steam in, $y 1$ is the master controlled variable, $y 2$ is the slave controlled variable,
TABLE 2: Fuzzy rule base $\left(K_{\mathrm{FP}}, T_{\mathrm{FI}}\right)$.

\begin{tabular}{lcccc}
\hline Error\gain & & Low $(\mathrm{L})$ & Medium $(\mathrm{M})$ & High $(\mathrm{H})$ \\
\hline Negative high & $\mathrm{NH}$ & & & $\mathrm{X}$ \\
Negative medium & $\mathrm{NM}$ & & $\mathrm{X}$ & \\
Zero & ZR & $\mathrm{X}$ & & \\
Positive medium & PM & & $\mathrm{X}$ & \\
Positive high & PH & & & $\mathrm{X}$ \\
\hline
\end{tabular}

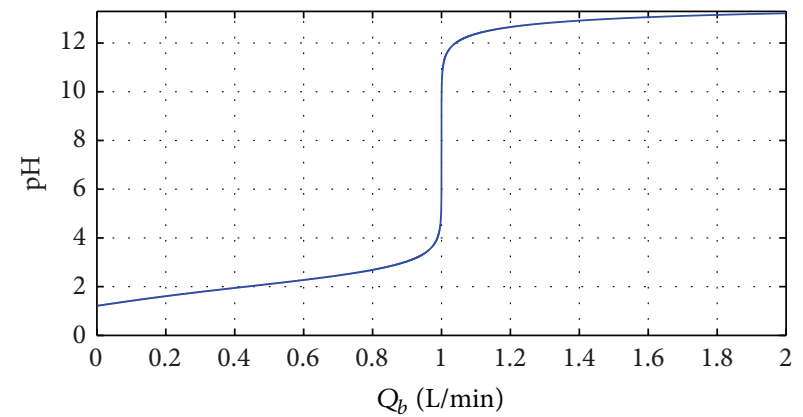

FIGURE 6: Titration curve of strong acid-strong base system.

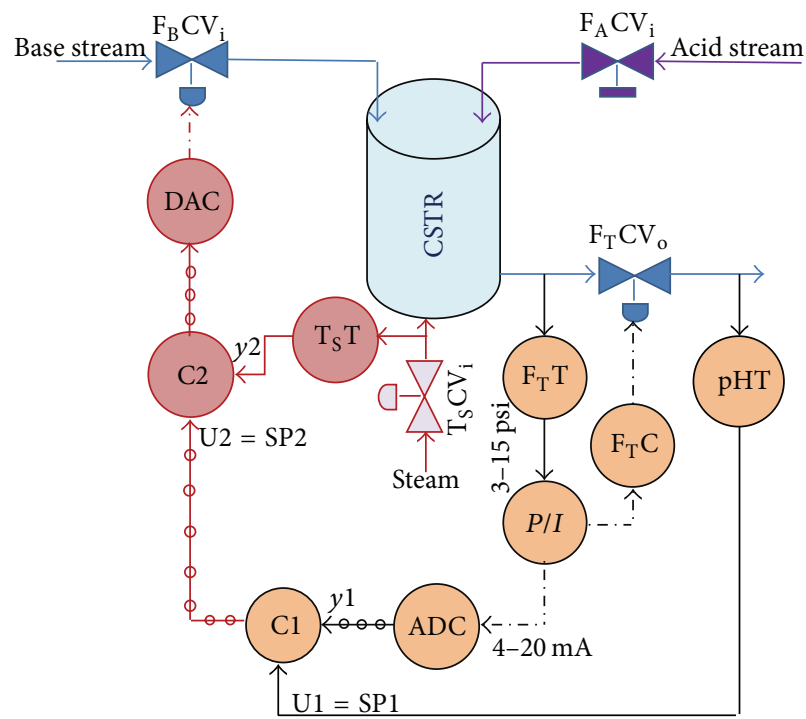

FIGURE 7: Schematic diagram of intelligent-based cascade control of $\mathrm{pH}$ reactor (CSTR).

$\mathrm{U} 2$ is the automatic setpoint of the slave controller, and $\mathrm{U} 1$ is measured setpoint of the master controller.

3.2.1. Fuzzy PI-Based Outer Loop (C1). The outer controller has been chosen to be accurate but slower than inner controller and designed to enhance the stability of the overall dynamic response of the $\mathrm{pH}$. The structure of the proposed fuzzy PI $(\mathrm{Cl})$ is shown in Figure 8.

The goal of the proportional gain in fuzzy system $\left(K_{\mathrm{FP}}\right)$ is to maintain stability. But the integral gain $\left(K_{\mathrm{FI}}\right)$ is used to ensure low oscillations.

Table 2 shows the fuzzy rule base for proportional gain $K_{\mathrm{FP}}$ and integral time $T_{\mathrm{FI}}\left(=1 / K_{\mathrm{FI}}\right)$ where five fuzzy 
TABLE 3: The integral of the absolute error (IAE) for the control methods.

\begin{tabular}{|c|c|c|c|}
\hline \multicolumn{4}{|c|}{ IAE } \\
\hline Process & Disturbance & $\begin{array}{l}\text { MPNN controller } \\
\text { (cascade structured) }\end{array}$ & $\begin{array}{c}\text { Fuzzy PI } \\
\text { (single feedback) }\end{array}$ \\
\hline \multirow{4}{*}{$\mathrm{pH}$ reactor } & Acid stream flow rate & 3.333 & 17.650 \\
\hline & Acid stream flow rate & 5.077 & 20.656 \\
\hline & Setpoint & 8.598 & 14.190 \\
\hline & Setpoint & 9.022 & 15.169 \\
\hline
\end{tabular}

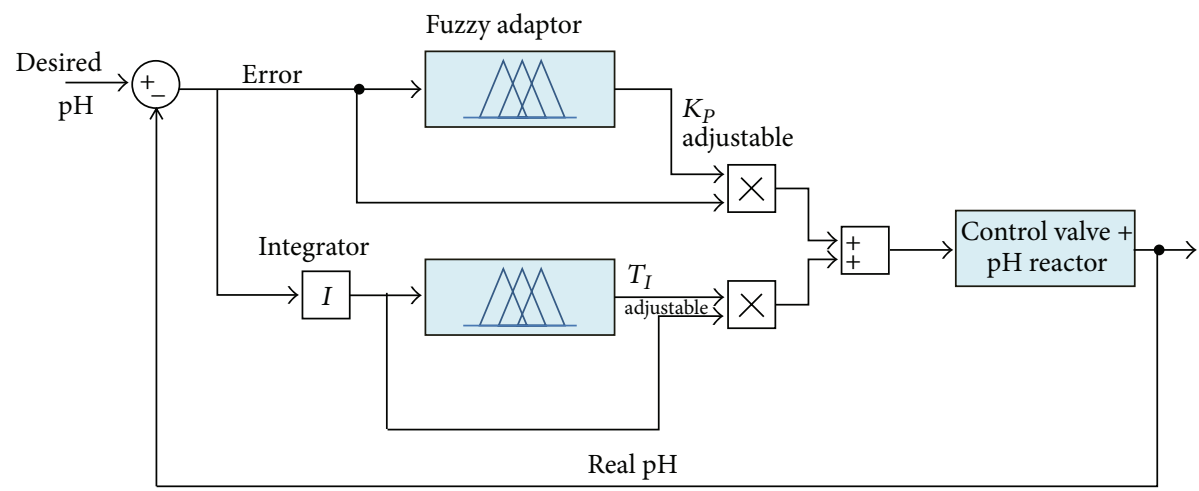

FIgURE 8: The structure of PI controller with fuzzy adaptation (outer loop).

triangular sets were considered for the error as a measure of the difference between the process output and its reference trajectory. Likewise, three triangular sets were considered for the output variables. For each case, five fuzzy rules were considered (Table 2).

3.2.2. MPNN-Based Inner Loop (C2). The MPNN has been proposed in the inner loop to be faster than outer one (fuzzy PI-based one). It aims to modify the dynamics of the overall response of the plant and to upgrade the reference trajectory (SP2) of the slave controlled variable $(y 2)$. The NN plant model is trained using the Levenberg-Marquardt algorithm. The training data were obtained from the nonlinear model of the $\mathrm{pH}$ reactor (see (3), Figure 6). The predictions are used by a numerical optimization program to determine the control signal that minimizes performance criterion over the specified horizon. The controller block was implemented in Simulink. Constraints and parameters values are $0 \leq u \leq$ $0.02,354 \leq y_{p} \leq 365, N_{1}=1, N_{2}=7, N_{u}=3$, and $\lambda=0.5$.

\section{Results}

Figure 9 shows predicted plant output using predictive neural network when comparing with the real plant one.

It is clear that the performance of the predictive neural network control (PNNC) is robust enough, since the predicted NN output well follows the real plant output referring to the same input function. The prediction error between the plant output and the NN output is used as the neural network training signal (Figure 3). Also, a random reference signal (as input function) is connected to the optimization block in the PNNC.
Figure 10 demonstrates the simulation results obtained from implementation of the design shown in Figure 7.

And hereby, to validate our design, the cascaded structure was compared with single feedback control structures, and the results are as given in Figure 11.

It has well been noted that the cascade MPNN-based structure (Figure 10) provides enhanced stability and modified dynamics when comparing with conventional (singlefeedback) intelligent-based one, Figure 11.

The simulation results in Figures 10 and 11 are achieved at two operational modes of $\mathrm{pH}$ reactor, acidic solution when $\mathrm{pH}$ is equal to 13 and basic one when $\mathrm{pH}$ is equal to 4.5. And so, the nonlinear regions in the titration process and the linear ones, seen in Figure 5, are covered. Both cases aim to validate the proposed control philosophy (predictive NNbased cascade control) at two extreme and opposite operating conditions.

\section{Interpretation of Results}

The quantitative performance values, integration of absolute error (IAE), for the MPNN in cascade design and fuzzy PI controllers are calculated as given in

$$
\mathrm{IAE}=\int_{0}^{T}|e| \cdot d t .
$$

The control performance of $\mathrm{pH}$ has been verified once with injection of acid stream flow rate as disturbance (rows 2 and 3 ) and also with submitting a nonstationary setpoint (rows 4 and 5).

It is well noted from Table 3 that when computing the IAE with single feedback design, it is found (as an example = 

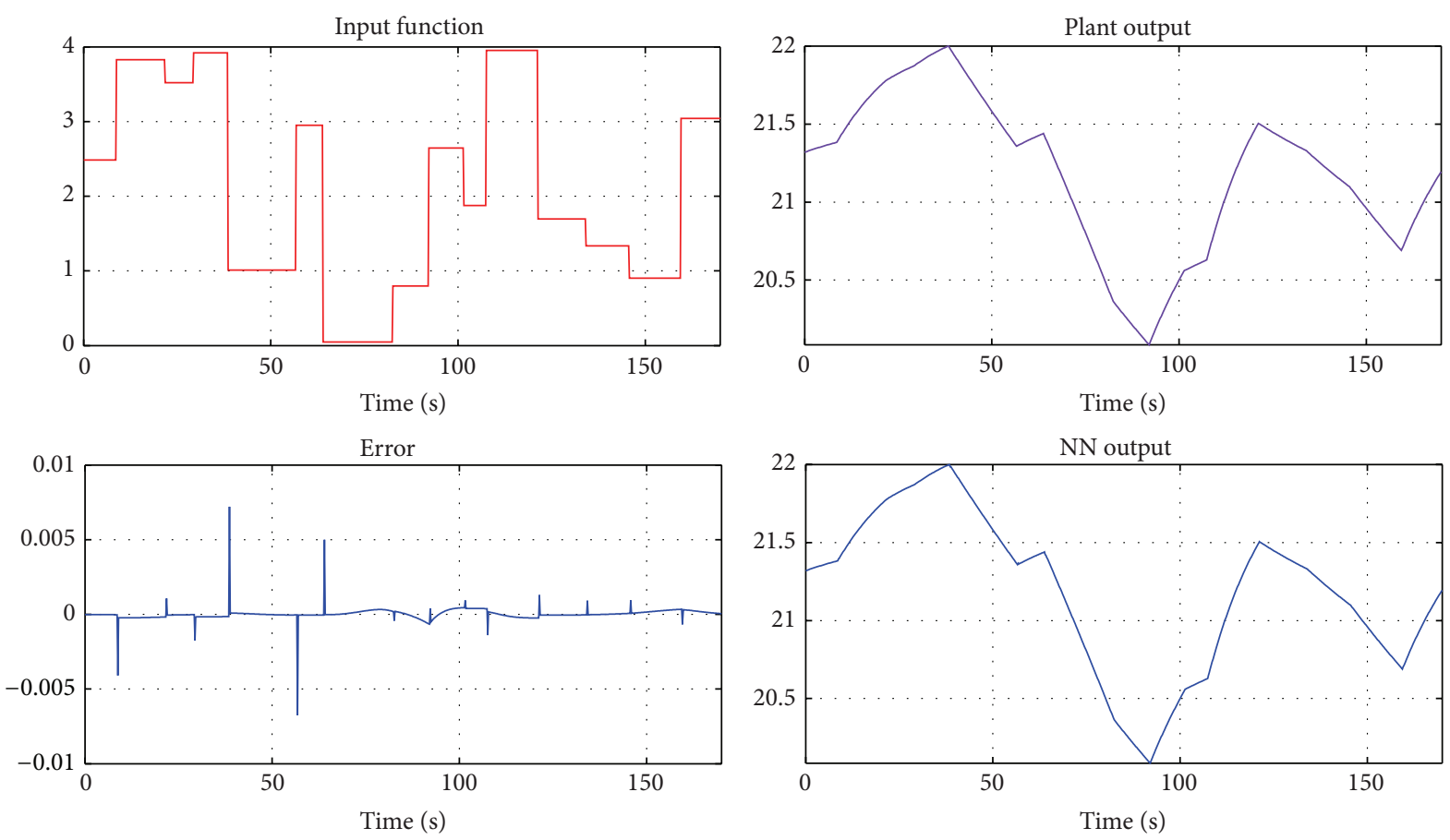

FIGURE 9: Performance of model-based predictive NN.

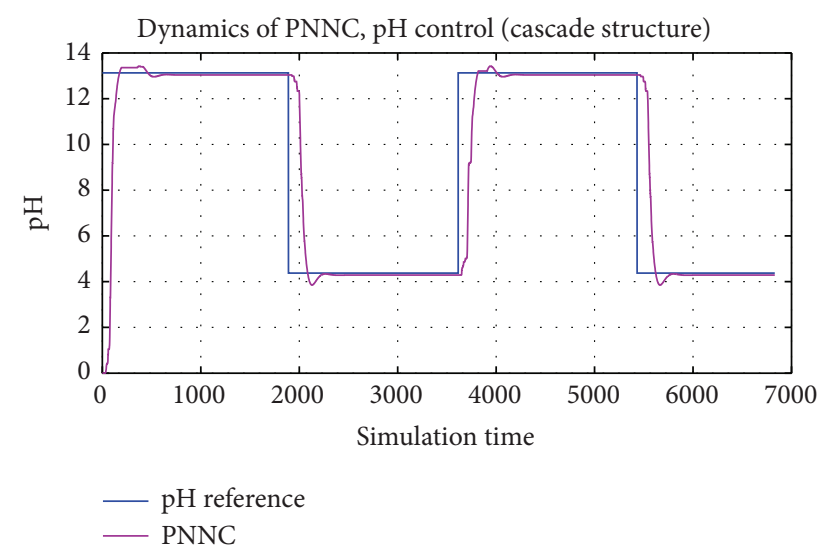

FIgure 10: Dynamics of PNNC with cascade structure.

17.650) but for cascade structure it is equal to 3.333. So, it could be concluded that the single feedback design (column 4) is worse than that of cascade structure (column 3).

\section{Conclusions and Future Work}

6.1. Conclusions. This paper has demonstrated the usefulness and effectiveness of applying neural networks in a modelbased control strategy to control a $\mathrm{pH}$ reactor.

Both the model predictive neural network as a master control loop and fuzzy PI controllers as slave one have been implemented in cascade structure (Figure 6). The controller performance under multiple changes in setpoint as well as the effect of fluctuation in acid stream as a disturbance has been investigated.

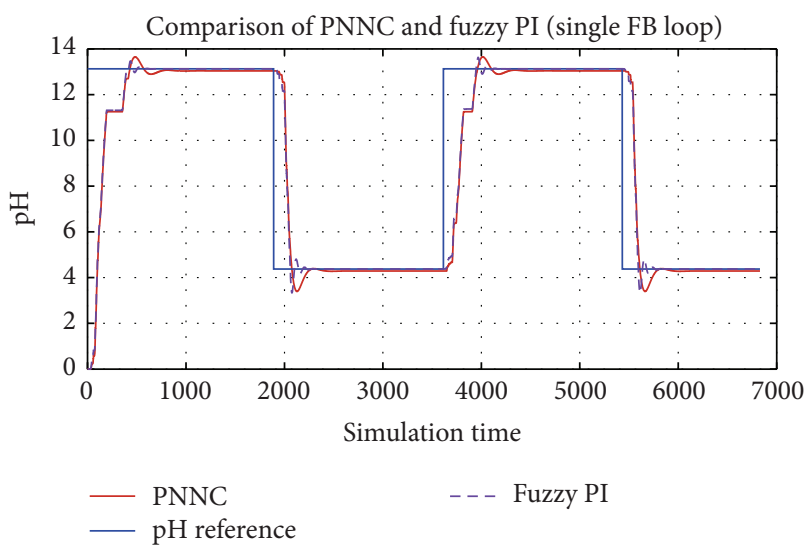

Figure 11: Dynamics of cascade structure of MPNN and fuzzy PI with convenient fuzzy PI one.

Comparison of performance with the conventional single feedback fuzzy PI controller indicated that cascade structure was more robust than the single feedback controller and gave better results in cases involving disturbances (Figures 10 and 11, Table 3).

Presented results confirm the known fact, with the chemical $\mathrm{pH}$ reactor, and when disturbances affect the control input, the cascade control structure can improve control performance in transient and steady-state conditions.

\subsection{Suggestions for Future Work}

(i) A rational controller could be added to manage the percentage in variation of base stream with respect to 
acid stream (e.g., weak or strong). Thus, the dynamics shown in Figure 10 can be further modified.

(ii) The proposed cascade structure and predictive NNbased controller could be realized using digital signal processor (DSP).

\section{Competing Interests}

The authors declare that they have no competing interests.

\section{Acknowledgments}

This project was supported by the Deanship of Scientific Research at Prince Sattam bin Abdulaziz University under the research Project no. 2016/01/6637.

\section{References}

[1] K. Rupinder, A comparison of different controllers to control various parameters of continuous stirred tank reactor [M.S. thesis], Thapar University, Punjab, India, 2012, Submitted in partial fulfillment of the requirements for the award of degree of master of engineering in Electronic Instrumentation and Control Department of Electrical and Instrumentation Engineering.

[2] A. Rani, V. Singh, and J. R. P. Gupta, "Development of soft sensor for neural network based control of distillation column," ISA Transactions, vol. 52, no. 3, pp. 438-449, 2013.

[3] A. Canakci, T. Varol, and S. Ozsahin, "Analysis of the effect of a new process control agent technique on the mechanical milling process using a neural network model: measurement and modeling," Measurement, vol. 46, no. 6, pp. 1818-1827, 2013.

[4] N. Ebrahimi and A. Gharaveisi, "Optimal fuzzy supervisor controller for an active suspension system," International Journal of Soft Computing and Engineering, vol. 2, no. 4, p. 36, 2012.

[5] I. M. Mujtaba and M. A. Hussain, Application of Neural Network and Other Learning Technologies in Process Engineering, Imperial College Press, London, UK, 2001.

[6] A. Arunyaand and A. S. Gandhi, "Design of cascade control system based on sustained oscillation," International Journal of Engineering Research \& Technology, vol. 3, no. 1, p. 1566, 2014.

[7] E. E. Ekpo and I. M. Mujtaba, "Evaluation of neural networksbased controllers in batch polymerisation of methyl methacrylate," Neurocomputing, vol. 71, no. 7-9, pp. 1401-1412, 2008.

[8] D. C. Psichogios and L. H. Ungar, "Direct and indirect model based control using artificial neural networks," Industrial \& Engineering Chemistry Research, vol. 30, no. 12, pp. 2564-2573, 1991.

[9] A. Y.-D. Tsen, S. S. Jang, D. S. H. Wong, and B. Joseph, "Predictive control of quality in batch polymerization using hybrid ANN models," AIChE Journal, vol. 42, no. 2, pp. 455-465, 1996.

[10] M. L. Thompson and M. A. Kramer, "Modeling chemical processes using prior knowledge and neural networks," AIChE Journal, vol. 40, no. 8, pp. 1328-1338, 1994.

[11] I. Al-Adwan, M. Al Khawaldah, S. Asad, and A. Al Rawashdeh, "Design of an adaptive fuzzy-based control system using genetic algorithm over a $\mathrm{pH}$ titration process," International Journal of Research and Reviews in Applied Sciences, vol. 17, no. 2, p. 177, 2013.
[12] S. Asad, "Observing of $\mathrm{pH}$ for titration process with hybrid neural network structure," International Journal of Research and Reviews in Applied Sciences, vol. 10, no. 2, pp. 264-271, 2012.

[13] S. Alsabbah, M. Aldhaifallah, and M. Al-Jarrah, "Design of multiregional supervisory fuzzy PID control of $\mathrm{pH}$ reactors," Journal of Control Science and Engineering, vol. 2015, Article ID 396879, 9 pages, 2015. 


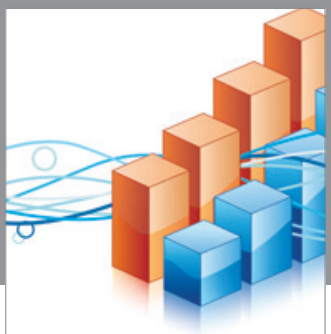

Advances in

Operations Research

vatem alat4

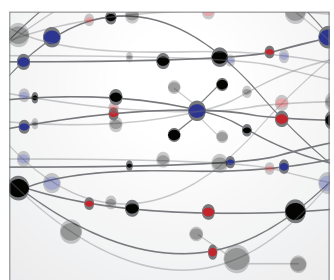

\section{The Scientific} World Journal
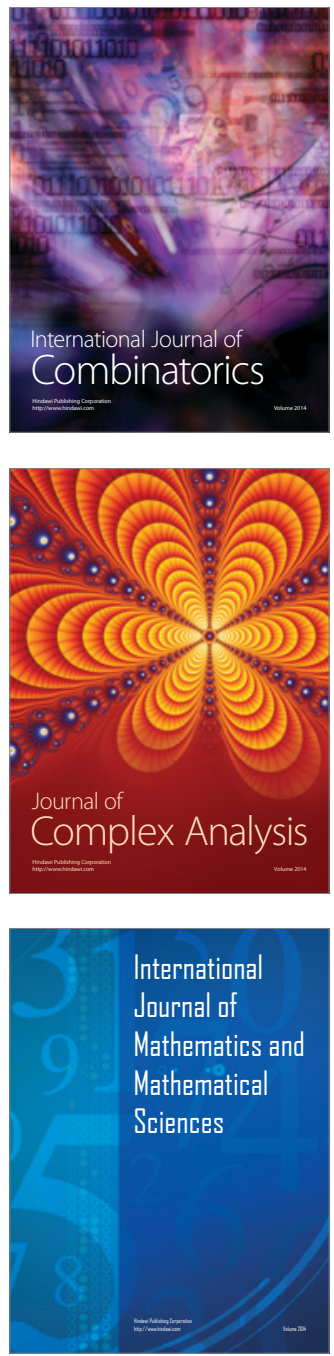
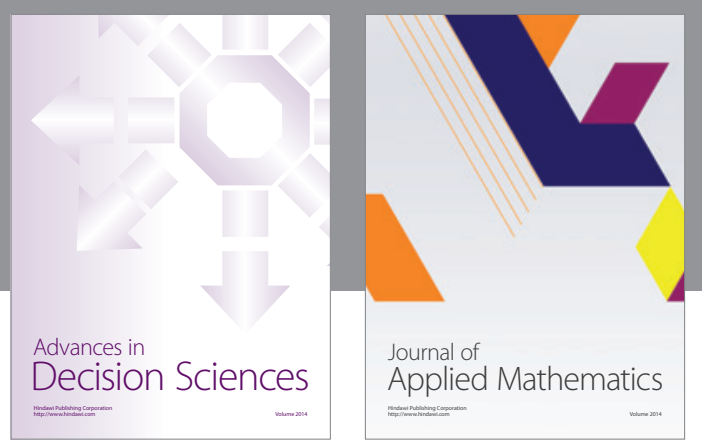

Algebra

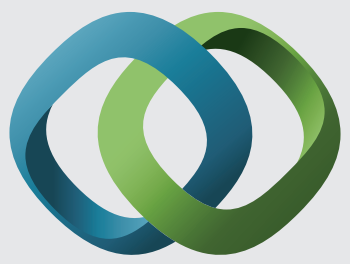

\section{Hindawi}

Submit your manuscripts at

http://www.hindawi.com
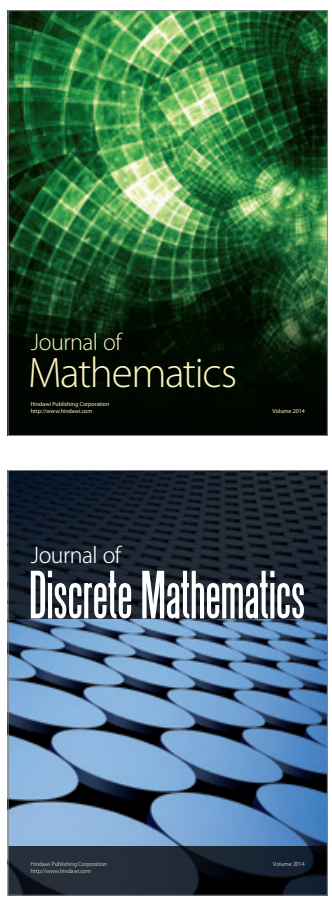

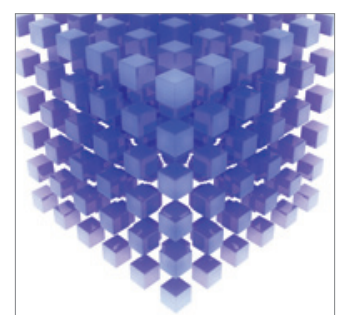

Mathematical Problems in Engineering
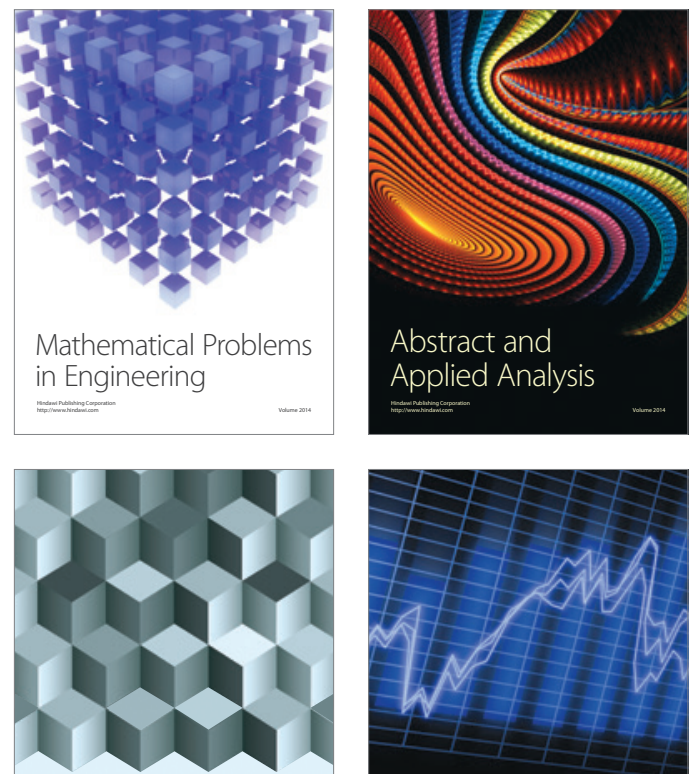

Journal of

Function Spaces

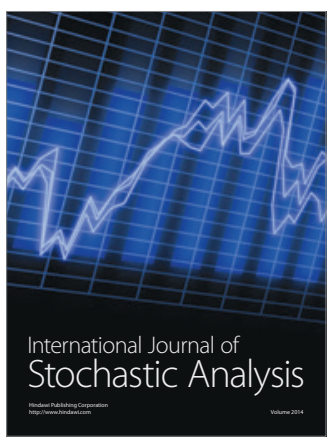

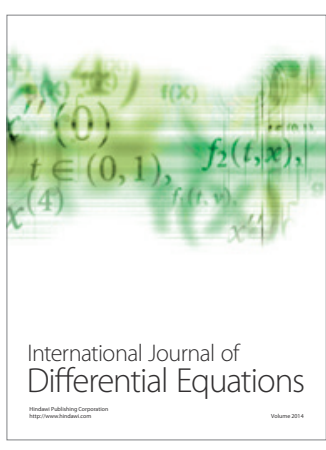
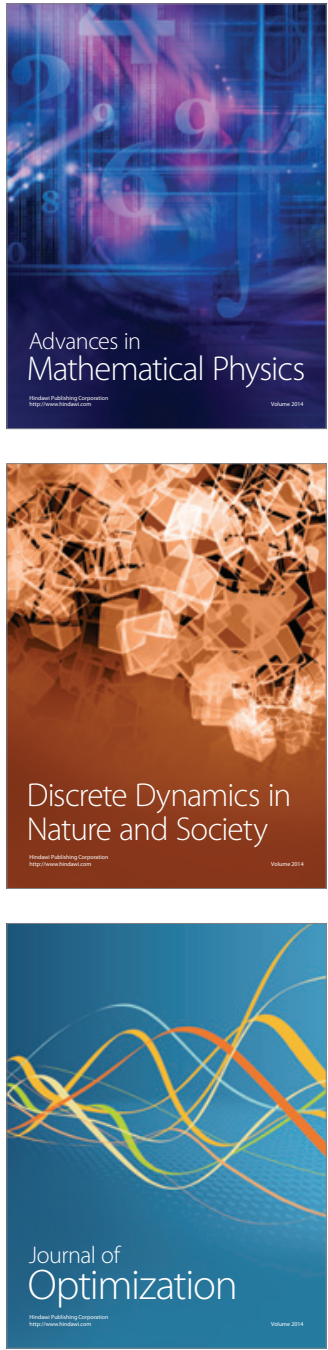